\title{
A simple and reliable procedure for preparing frozen sections of free mouse blastocysts
}

\author{
G. P. Schneider II* \\ Department of Anatomy, University of Michigan, Ann Arbor, Michigan 48104, U.S.A.
}

Certain histochemical and radioautographic procedures require frozen tissue sections for maximum efficiency. The procurement of frozen sections of mammalian blastocysts has been limited to the sectioning of frozen uteri at the proper stage of pregnancy. When fresh or frozen thin mounts of preimplantation embryos have been needed, squash techniques of the whole embryos have been used (Prasad, Dass \& Mohla, 1968; Sanyal \& Meyer, 1969). This communication describes a method whereby free blastocysts can easily and quickly be embedded and cryostat sectioned in the absence of all organic solvents with minimum disruption of the embryos.

Groups of 25 Swiss-Webster mouse blastocysts were fixed in $2 \mathrm{ml}$ neutral buffered formalin at $4^{\circ} \mathrm{C}$ for $1 \mathrm{hr}$. They were then transferred with a minimum of fixative into $1 \mathrm{ml} 10 \%$ gelatin in a trans-illuminated watch glass warmed to $37^{\circ} \mathrm{C}$. The watch glass was warmed by fitting it to the open end of a hollowed Perspex block through which water at $55^{\circ} \mathrm{C}$ was passed (Text-fig. 1). The

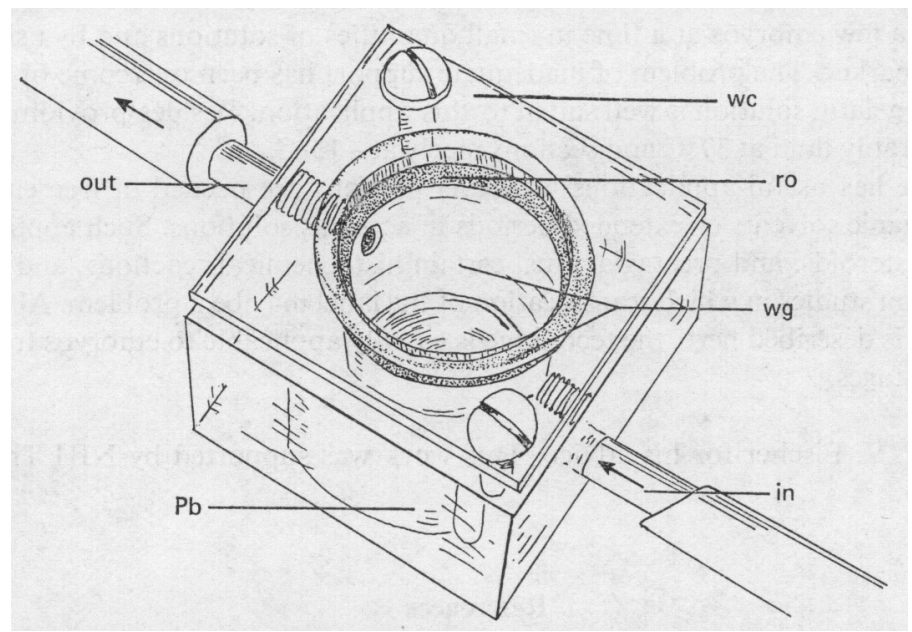

Text-fig. 1. Apparatus used to keep the gelatin solution warm for blastocyst transfer. Slowly flowing $55^{\circ} \mathrm{C}$ water is passed into (in) and out of (out) the hollowed chamber of the Perspex block (Pb), keeping the solution in the watch glass (wg) at a constant $37^{\circ} \mathrm{C}$. The watch glass was held tightly to the open end of the water chamber by the watch glass clamp (wc) and a rubber ' $O$ ' ring (ro). $\times 0.75$.

transfer of the blastocysts was precisely controlled by means of a $0 \cdot 2-\mathrm{ml}$ micrometer syringe attached by plastic tubing to a melting-point capillary tube drawn out to a diameter of $150 \mu \mathrm{m}$ at one end. Subsequent location of the blastocysts in the blocks and sections was aided by the addition of a few grains of water-insoluble carmine red powder (Carmin-Alum Lake: Hartman-Leddon Company) to the $10 \%$ gelatin. Before transfer of the blastocysts, approximately $0.1 \mathrm{ml} 10 \%$ gelatin without the carmine red was drawn into the transfer pipette to prevent the later expulsion of air bubbles. Then the blastocysts in the $10 \%$ gelatin and carmine red were injected and briefly stirred into a small drop of previously warmed $25 \%$ gelatin that was just beginning to solidify on a $1 \times 3$ in microscope slide. The drop of gelatin with the blastocysts was solidified by cooling the slide on an ice cube before quickly freezing it in a solid $\mathrm{CO}_{2}$-isopentane slush.

* Present address: Department of Anatomy, Michigan State University, East Lansing, Michigan 48824, U.S.A. 
The frozen drops of gelatin containing the embryos were trimmed to 3- $\mathrm{mm}^{2}$ blocks. After carefully removing these blocks from the slides, they were frozen to cryostat specimen holders with a small amount of cryostat embedding matrix. Serial sections were cut at $8 \mu \mathrm{m}$ from the blocks with a cryostat (International Equipment Company) and mounted on acid-cleaned $22-\mathrm{mm}^{2}$ cover slips. The cover slips were immediately placed on a $35^{\circ} \mathrm{C}$ slide-warming tray to dry and then soaked in distilled water for $5 \mathrm{~min}$ to remove the gelatin matrix, thus minimizing background staining. The soaking does not remove the blastocyst sections from the cover slips as long as the latter are very clean.

The technique is not difficult or time consuming. Each group of up to 25 blastocysts takes from 15 to $30 \mathrm{~min}$ to embed and much of the time is spent concentrating the embryos and carmine red grains at each transfer. The most difficult aspect is transferring the blastocysts into the $25 \%$ gelatin drop before it gels.

Fixation is not absolutely necessary in preparing the blastocysts for sectioning. However, the unfixed blastocyst is more likely to collapse away from the zona pellucida with the subsequent loss of morphological detail. The presence or absence of fixation has no effect on the ease of sectioning.

Most embedding and sectioning methods entail a high probability of the loss of small tissues such as isolated blastocysts. To circumvent this problem various procedures have been described (Metz, 1921; Samuel, 1942; Mintz, 1971). However, all have been applied to paraffin wax-embedding techniques and are not applicable when frozen sections are required since the tissues have no supporting matrix until embedded. In the method described here, not only can frozen sections be made without organic solvents and time-consuming rinses, but the problem of loss is kept to a minimum by handling only a few embryos at a time in small quantities of solutions and by using the carmine red powder as a marker. The problem of inadequate support has been overcome by the use of $25 \%$ gelatin. The $25 \%$ gelatin solution is well suited to this application. Besides providing the necessary support, it is workably fluid at $37^{\circ} \mathrm{C}$ and sections nicely at $-15^{\circ} \mathrm{C}$.

The procedure has useful applications whenever sections are needed of free embryos without the use of any organic solvents or extended periods in aqueous solutions. Such applications include studies on lipids, steroids, and prostaglandins, certain histochemical reactions, and any radioautographic localization studies in which translocation of the label may be a problem. Although only the use of blastocysts is described here, the technique is equally applicable to embryos from the one-cell to the blastocyst stages.

I thank Dr T. V. Fischer for his advice. This work was supported by NIH Training Grant 5 Tol GM 00312-13.

\section{References}

Metz, C.W. (1921) A simple method for handling small objects in making microscopic preparations. Anat. Rec. 21, 373-374.

Mintz, B. (1971) Allophenic mice of multi-embryo origin. In Methods in Mammalian Embryology, pp. 186-214. Ed. J. C. Daniel, Jr. W. H. Freeman and Company, San Francisco.

Prasad, M.R.N., Dass, C.M.S. \& Mohla, S. (1968) Action of oestrogen on the blastocyst and uterus in delayed implantation. An autoradiographic study. J. Reprod. Fert. 16, 97-104.

SAMueL, D.M. (1942) The use of an agar gel in the sectioning of mammalian eggs. $J$. Anat. 78, 173175.

Sanyal, M.K. \& MeYer, R.K. (1969) Deoxyribonucleic acid synthesis in vitro in preimplantation blastocysts of prepuberal rats ovulated with gonadotrophins. Endocrinology 85, 585-589.

Received 16 June 1975 